\title{
Studying the anonymity trilemma with a discrete-event mix network simulator
}

\author{
Ania M. Piotrowska \\ Nym Technologies
}

\begin{abstract}
In this work, we present a discrete event mix network simulator, which allows analysing how anonymity, latency, and bandwidth overhead are affected by various scenarios of deployment and design choices. These design choices include network topology, mixing technique, volume of traffic, latency requirements, packet size or use of cover traffic. To the best of our knowledge, this is the first such simulator as work on it began in 2017 to analyze the Loopix mix network, and the code of our simulator is available under an open-source license. To demonstrate the capabilities of our simulator, we perform an empirical analysis of the impact of core design choices on anonymity, scalability and latency in Elixxir, HOPR and Nym, currently deployed mix network infrastructures that make a variety of different choices in their design.
\end{abstract}

\section{INTRODUCTION}

Network traffic is a source of privacy-sensitive information, both in terms of the content and metadata. Encryption is only the first step in the efforts to achieve privacy in the online world, since it can only protect the confidentiality of our communication, but does not hide the unique characteristics associated with the communication, the so-called metadata. Exposing the metadata not only undermines the properties provided by the use of encryption $[5,9,10,18,20,42$, $49,50]$, but also can be exploited to uniquely identify users, enable pervasive tracking, and reveal private information about personal or business activities [6, 14, 24, 29, 31, 32, 45, 46].

Anonymous communication networks hide the network metadata and the relationship between communicating parties on the Internet. Since 2004, Tor has become the most popular anonymous communication tool, attracting at least 2 million users daily. However, as research has shown [35, 36, 38-41, 51] Tor offers limited security guarantees against traffic analysis techniques. For that reason, over the past years, there has been an increased interests in mix network technologies, with companies like Elixxir, HOPR or Nym collectively attracting millions in investment [30] with widely contrasting designs.

While great strides have been made, a key obstacle to the real world deployment of mix networks is that it is difficult to tune the trade-offs between privacy and network performance in a given design. Therefore, we present a discrete event mix network simulator, which allows us to empirically evaluate the privacy and performance properties of various mix network designs via simulation. In particular, our simulator allows analysing how anonymity, latency, and bandwidth overhead are affected by various scenarios of deployment of such networks and design choices. We next use the presented simulator to investigate how anonymity and end to end latency are affected by user loads, network topology, and mixing strategies on the example of Elixxir [2], HOPR [4] and Nym [1]. To the best of our knowledge, this is a first such empirical analysis of those networking designs.

\section{BACKGROUND}

The concept of an anonymous communication network was pioneered by Chaum [7] to mitigate the threats of progressive communications surveillance. Their main security goal is to shield the correspondence between senders and recipients by hiding the distinctive characteristics of the network traffic, the so called metadata. The principal concept of the Chaum's anonymous communication network is the decentralized mix network in which the network traffic is relayed via a set of independently operated cryptographic relays called mix nodes. In contrast to onion routing [23], however, mixnets route each individual packet via independent routes. By routing the traffic through a sequence of independent nodes, mix networks protect users against disclosing their identity to end receivers.

In order to ensure anonymous communication, the traffic is layer encrypted using public-key cryptography. The encapsulation of the packets into a special cryptographic packet format provides the bitwise unlinkability between the bit patterns of the encoded messages entering and decoded ones leaving the mix node. This ensures that any third-party observer is not able to trace the packets based on their binary representation. Moreover, malicious nodes which route the packets cannot eavesdrop on user traffic, as none of the mix nodes knows the whole connection, they only know the previous and next hop, therefore only the first mix knows who is the sender, and the last mix knows who is the recipient.

In addition to transforming the routed packets cryptographically, the nodes perform the reordering (mixing) operation so that the incoming and outgoing packets cannot be linked based on timing. Mixnets thus provide strong metadata protection against an adversary that can observe the entire network and perform traffic analysis attacks.

Originally, Chaum proposed to relay all packets through a fixed set of mix nodes, called a cascade. The mixes used the batch-andreorder mixing technique, in which a mix collects a certain threshold of packets, decrypts them and shuffles lexicographically, and finally forwards to the next mix in the cascade. However, the fixed cascade topology scales poorly, since once the cascade reaches its maximum capacity you cannot support more traffic, and this often introduces reliability issues and congestion in packets transmission. Moreover, the end-to-end latency of packets is unbounded since one cannot predict in which batch their packet will be accepted. Therefore, although they offered strong anonymity the early deployments of the original Chaumian mixnets have become unfashionable due to a perceived higher latency that cannot accommodate real-time communications. 
Over the last two decades, there were significant efforts $[11,22$, $28,34,52,53]$ to tackle the scalability, privacy, and latency problems of the early designs anonymous communication systems. In particular, companies like Elixxir, Nym and HOPR focus on developing novel mix network infrastructures to provide anonymous communication. Although all of them develop source-routed decryption mix networks, their key design choices differ regarding topology, mixing technique and onion encryption.

Elixxir [19], a communication infrastracture used by the $\mathrm{xx}$ network [3], implements a variant of the cMix protocol [8]. Like the traditional Chaumin mixnet [7], Elixxir uses the batch-andreorder mixing technique. In order to tackle the problem of timeconsuming public key operations and in order to accelerate the packets' processing, Elixxir introduces a precomputation phase, which allows to perform the public key operations before the realtime phase of handling messages between senders and recipients. In result, during the real-time phase, mixes cryptographically process the received packets using the shared values established during the precomputation. However, the time needed to perform the precomputation phase grows linearly with the size of the anonymity set, and it has to be repeated before each real-time communication phase.

The nodes in the network are grouped into small ephemeral teams, each one forming a cascade topology. At any given time there might be multiple teams, in varying stages of precomputation phase, only one team is responsible for transporting the network packets in real-time. Once the batch processing is completed, the team disbands and the nodes can be placed in a new random team. In other words, currently cMix uses a single cascade topology. In the future it might be replaced with a multiple cascade topology, in which several cascades work in parallel, to handle the increasing traffic.

Nym [13] network is underpinned by the Loopix design [44] The Nym mixes are grouped into layers which form a stratified topology [16]. Each mix in layer $i$ is connected with each mix in the previous and next layer, and traffic flows from the first to the last layer. According to Nym's open-source code base, the current topology has three layers. In contrast to the original Chaumian mixnet or Elixxir, Nym uses a variant of continues-time mixing technique [27], in which a mix delays each packet before forwarding it to the next hop. The amount of time a packet needs to wait in each mix is chosen by the sender, who picks it at random from an exponential distribution.

In order to circumvent the time-consuming public key operations used for onion encryption, Nym uses the Sphinx cryptographic packet format [12]. Sphinx is a compact and efficient cryptographic packet format, that provides bitwise unlinkability for multi-hop routing and supports features like anonymous replies, resistance to tagging attacks, and hiding of the routing information.

HOPR is building a peer-to-peer based network for anonymous messaging [26, 47], where participants act both as relay nodes and end users. Similarly to Nym, packets are encapsulated using Sphinx and routed through a sequence of few intermediaries to a receiving peer. ${ }^{1}$ It is not yet clear which mixing technique HOPR peers will use. According to the whitepaper [47], HOPR is developing a Chaumian mixnet. However, the mixing technique currently implemented works differently from the one used in a Chaumian mix. ${ }^{2}$ Instead of batching and shuffling a fixed number of packets, a HOPR node adds the incoming packets to a single queue from which it picks uniformly at random one packet at the time to process and sends it to the next hop.

\section{MIX NETWORK SIMULATOR}

We developed an open-source mix network simulator using Pутноn3. The interactions between the different network components are simulated using the process-based discrete-event framework Simpy. In this paper, we evaluated our experiments using the AWS EC2 instances. The code of our simulator is available under an open-source license [43].

\subsection{Simulator configuration}

Network topology. Currently, our simulator supports four different network topologies, i.e., (1) cascade, (2) multi-cascade, (3) stratified and (4) peer-to-peer topologies. Further, we also allow for different configuration of the selected topology, including: total number of nodes (for cascade and p2p), number of parallel cascades (for multi cascades), and number of layers and nodes per layers (for stratified).

Mixing technique. Our simulator implements two mixing techniques, (1) the batch and reorder technique and (2) the Poisson mixing technique [44], which is a variant of the continuous time mixing technique [27].

Cover traffic. An important feature of anonymous communication networks is cover traffic, which can be deployed to ensure that there is sufficient traffic in the network to guarantee a large anonymity set, as well as, disguising communication patterns of the individual users. Our implementation, if desired, allows for simulating cover traffic generation by either clients or intermediate nodes. Additionally, the frequency of cover traffic generation can be adjusted accordingly.

Sending behaviour. In our simulation, the traffic generated by the end users is modeled by the Poisson distribution. We chose this distribution as it is appropriate if the arrivals are from a large number of independent sources, like in networks with many clients and nodes. Moreover, Poisson models have been widely used in computer networks and telecommunications literature [25, 37]. The configurable parameter of the Poisson distribution allows simulating different sending patterns of the clients depending on the use case. In addition to the above configurable parameters, our simulator implements also customisable packet and message sizes, and message fragmentation.

\footnotetext{
${ }^{1}$ According to the current code base, the number of hops is 1 but can also be userdefined.

${ }^{2}$ https://github.com/hoprnet/hoprnet/blob/d27a8c506491dc7788259616b9c81307a0ecff97/ packages/core/src/mixer.ts\#L25
} 


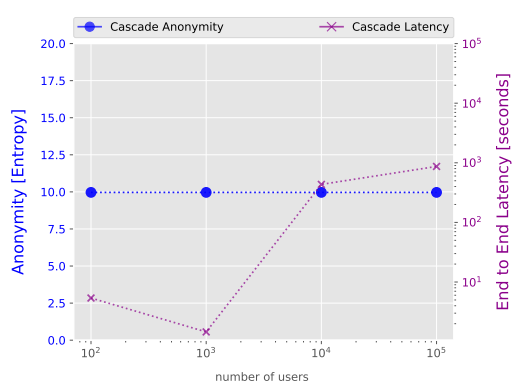

(a) Single cascade Elixxir network

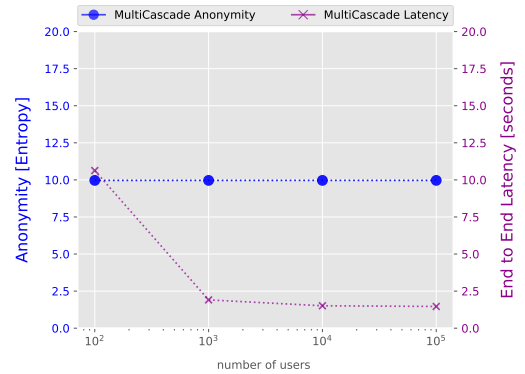

(b) Multi cascade Elixxir network

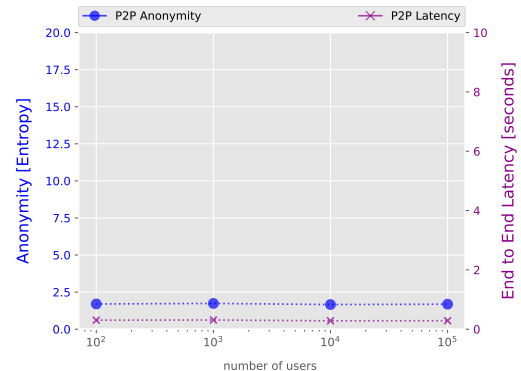

(c) HOPR mix network

\subsection{Anonymity metrics}

We currently implement the two following metrics to quantify privacy offered by the analysed network:

Entropy. A common measure of anonymity is the anonymity set, which reflects the number of other packets with which our message can be confused by the attacker. However, a global passive adversary monitoring the network can assign different probabilities for each outgoing packet being linked to the observed incoming packet. This can leak a lot of information to the attacker. Therefore, to measure the end to end anonymity we implement the metric based on Shannon entropy introduced in [48]. In a nutshell, each outgoing packet will have a distribution over being linked with past input packets, and the entropy of this distribution is our anonymity metric (see Appendix A). By using entropy, we gain a measure of how indistinguishable the packets shuffled by the mix network are among each other, hence how uncertain is the adversary about which of the outgoing packets is his target one.

Third-party unlinkability. Our simulator implements also the third-party unlinkability metric introduced in [44]. This metric measures the chances of the adversary correctly correlating the communicating parties even if they know in advance that either $S_{1}$ or $S_{2}$ are communicating with $R$. The metric measures the expected difference in likelihood that a given packet received by $R$ is sent from $S_{1}$ in comparison to $S_{2}$. In Appendix A we present the formal definitio of this metric and describe how do we quantify information leaked given multiple rounds of observation.

\section{EMPIRICAL STUDY OF ELIXXIR, HOPR AND NYM}

Using the simulator, we analyse the privacy (as measured in by entropy) and performance offered by mix network designs like Elixxir, HOPR and Nym. In particular, we take a closer look at how the core design decisions impact the scalability, anonymity and latency overhead.

Assumptions. In order to make our analysis fair, we make the following assumptions. For the HOPR and Nym networks we assume the same processing capacity of the mix nodes (i.e., single node has a capacity to process 1000 packets per second). Furthermore, in the case of Elixxir network we assume a batch of size 1000 packets Moreover, we exclude from our analysis in terms of end-to-end latency the time needed for the cryptographic processing of the

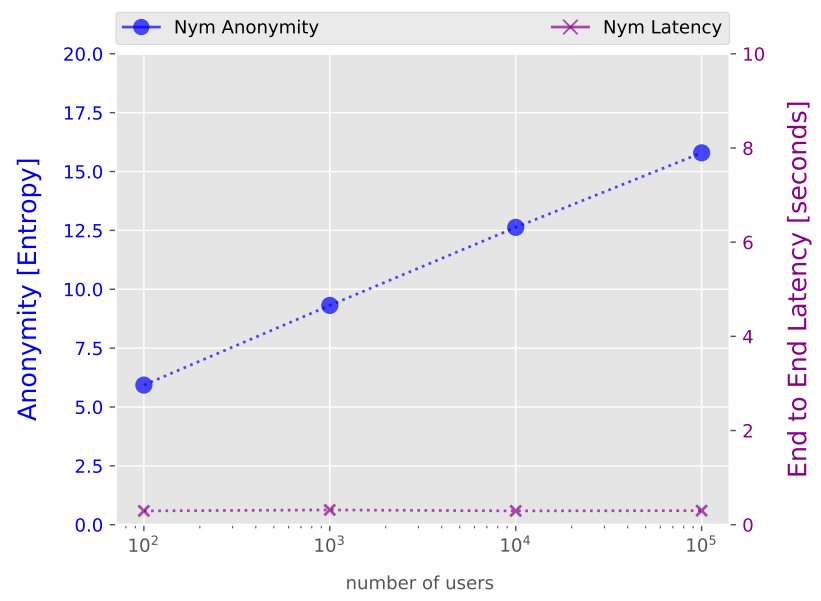

Figure 2: Nym: Impact of increasing user base on anonymity and end to end latency.

packets, as this time depends on the underlying onion encryption protocol, as well as the choice of the implementation language and the optimization of the code base. The end-to-end latency is given purely by the time needed to mix the packets.

Although HOPR and Nym implement different mixing techniques (see Section 2), from a theoretical standpoint the information leaked to the adversary observing a single mix node is the same. This is due to the fact that the exponential distribution used by Nym to generate the intermediate delays has the memoryless property [33], meaning that the probability that a packet leaves a mix at a given time is independent of its arrival time. Thus, from the perspective of the network observer each packet in the mix's pool has equal chances to be the next one to be forwarded. Therefore, in our simulation we use for both HOPR and Nym the Poisson mixing technique [44]. Without a loss of generality, unless stated otherwise, we assume the mean parameter of the exponential distribution to be 0.1 second per node. Similarly, we assume that each end user sends on average 1 packet per second following a Poisson process. Finally, all packets between end users are routed via 3 mix nodes.

Results. Anonymity loves company [15], hence scalability is one of the key properties of any anonymous communication network. As shown in Figure 1a mix networks which have a cascade topology 


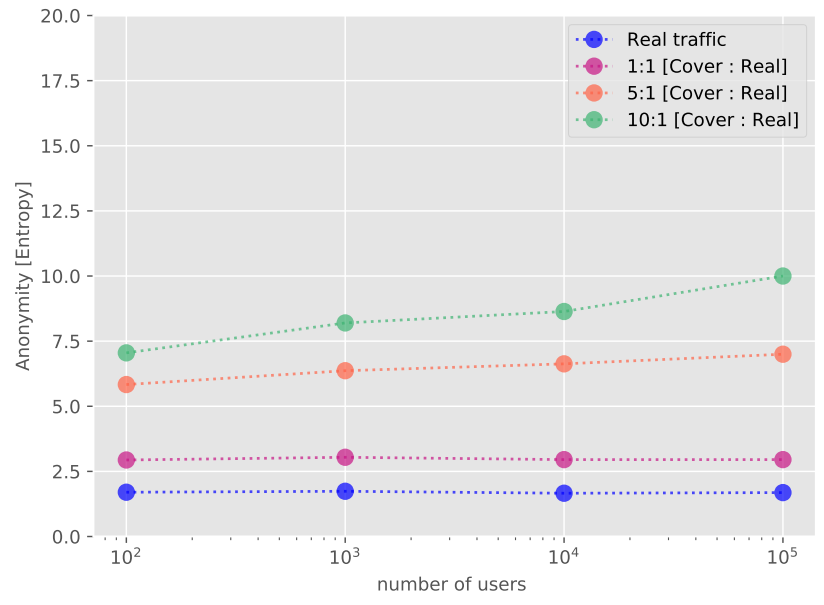

Figure 3: HOPR: Volume of cover traffic needed to provide desired levels of anonymity given increasing user base.

and batch-and-reorder mixing techniques (like Elixxir) scale poorly with an increasing user base. This design imposes large latency overhead when the volume of traffic grows, as the cascade becomes congested and each packet has to wait for a long period of time to be processed. Note that this design imposes high latency overhead not only when the volume of traffic is high but also when the traffic volume is low since each node waits a long time to collect a full batch for processing. Thus, end users have no control or prediction on the end-to-end latency of their packets.

An alternative approach, which significantly improves scalability is to introduce multiple cascades which work in parallel, a solution proposed by Elixxir. ${ }^{3}$ As shown in Figure 1b, such approach allows indeed the network to scale out and thus avoid a high latency overhead. However, the anonymity provided is still always constant as it is limited to the fixed size of the batch. Thus the mix network does not profit from the increasing number of users. Moreover, even with multiple cascades, Elixxir still imposes much higher end-toend latency that HOPR and Nym. As Figure 1c shows, networks like HOPR that combine a P2P topology with continuous-time mixing scale well but provide very poor anonymity even when there is an increasing number of users, because the user traffic is spread out thinly through the network. Moreover, since HOPR nodes select packets to forward randomly from the pool of currently stored packets, the end user has no guarantees on bounds for delivery time of sent packets, as is also the case of the batch and reorder technique.

On the other hand, as shown on Figure 2, the stratified topology used by Nym scales out by adding more nodes per layer to support a large user base without a negative impact on the end-to-end latency. In particular, the end to end latency is bound by the mean parameter of the exponential distribution used by the individual senders. ${ }^{4}$ Critically, the anonymity provided by Nym increases as

\footnotetext{
${ }^{3} \mathrm{~A}$ new cascade is added only once all of the existing ones are at their full capacity. ${ }^{4}$ In implementation, it would also be bound by the time of processing the cryptographic packet format used for encryption.
}

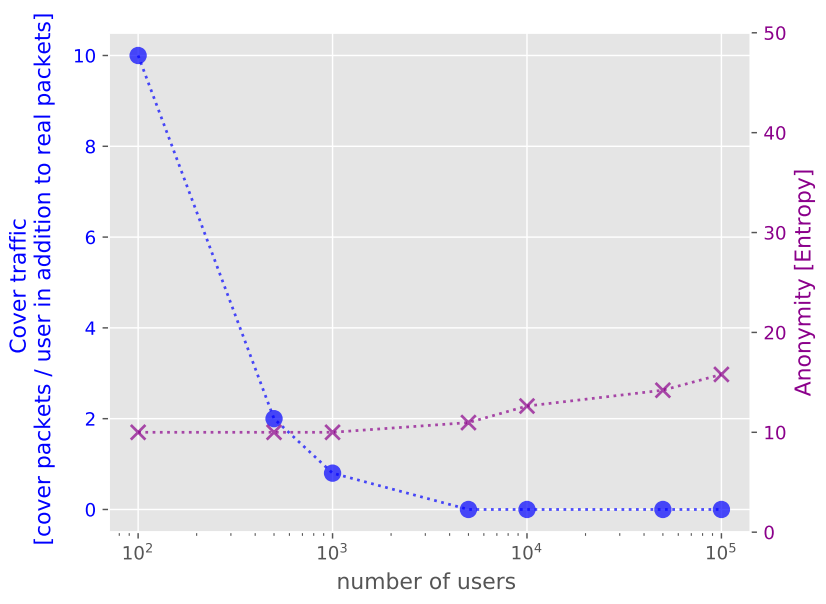

Figure 4: Nym: Volume of cover traffic needed to provide minimum desired anonymity 10 in entropy given increasing user base.

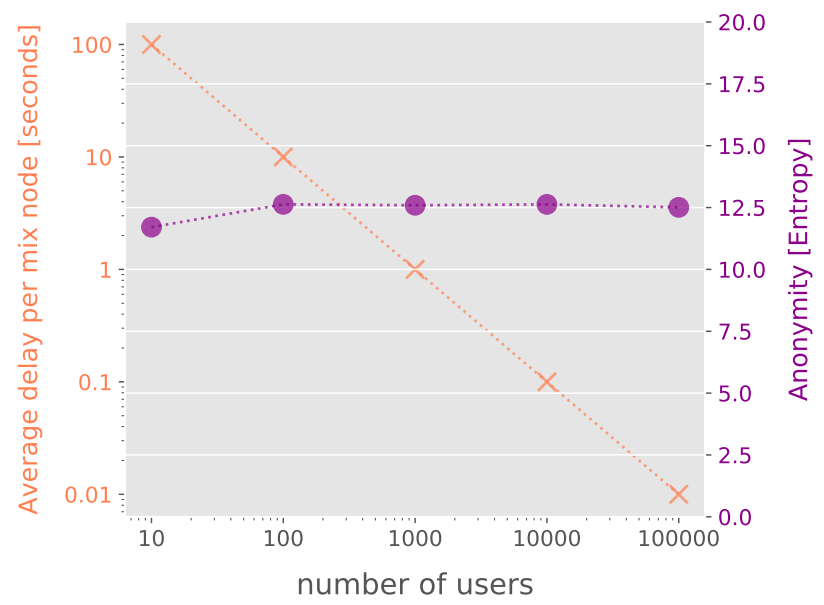

Figure 5: Nym: Impact of increasing volume of traffic on required mixing delay.

the network grows. The more traffic flows into the Nym mix network, the larger the anonymity as measured in entropy. This is due to the fact, that although the traffic flows via multiple independent routes, the connectivity of a stratified topology guarantees that the routes intersect, hence all packets contribute to the same anonymity set. In contrast to the multi-cascade Elixxir and HOPR, Nym provides much better anonymity for large volumes of users. What differentiates Nym from designs like Elixxir and HOPR is that the increasing number of Nym users allows to tune down the mean parameter of the exponential distribution, and thus decrease the end-to-end latency, without sacrificing anonymity (Figure 5).

One way to improve the level of anonymity is to deploy cover traffic. However, as our analysis shows (Figure 3), P2P networks like HOPR need huge volumes of cover traffic to reach reasonable 
levels of anonymity. In contrast, as Figure 4 depicts, once the number of users increases in the Nym mix network (and hence the volume of real packets flowing into the mix network), the amount of cover traffic needed to provide a constant desired anonymity decreases. Moreover, in contrast to HOPR in which the end users carry the burden (and cost) of generating the cover traffic, in Nym this responsibility can be delegated to the mixes.

\section{CONCLUSION}

In this paper, we outlined the design and implementation of a novel discrete-event mix network simulator, which can be used to investigate how different network parameters, design choices or user loads impact both the anonymity as well as the latency and the bandwidth overhead. We further use our simulator as a tool to compare three fast growing mix network infrastructure: Elixxir, HOPR and Nym.

\section{REFERENCES}

[1] 2019. Nym Technologies. https://nymtech.net

[2] 2019. xx network. https://xx.network/.

[3] 2019. xx network whitepaper. (2019). https://xx.network/xx-whitepaper-v1.3.pdf

[4] 2020. HOPR. https://hoprnet.org.

[5] Alireza Bahramali, Amir Houmansadr, Ramin Soltani, Dennis Goeckel, and Don Towsley. 2020. Practical Traffic Analysis Attacks on Secure Messaging Applications. In 27th Annual Network and Distributed System Security Symposium, NDSS 2020, San Diego, California, USA, February 23-26, 2020. The Internet Society. https://www.ndss-symposium.org/ndss-paper/practical-traffic-analysisattacks-on-secure-messaging-applications/

[6] Carole Cadwalladr and Emma Graham-Harrison. [n.d.]. Revealed: 50 million Facebook profiles harvested for Cambridge Analytica in major data breach. The Guardian ([n.d.]). https://www.theguardian.com/news/2018/mar/17/cambridgeanalytica-facebook-influence-us-election.

[7] David Chaum. 1981. Untraceable Electronic Mail, Return Addresses, and Digital Pseudonyms. Commun. ACM 24, 2 (1981), 84-88. https://doi.org/10.1145/358549. 358563

[8] David Chaum, Debajyoti Das, Farid Javani, Aniket Kate, Anna Krasnova, Joeri de Ruiter, and Alan T. Sherman. 2017. cMix: Mixing with Minimal Real-Time Asymmetric Cryptographic Operations. In Applied Cryptography and Network Security - 15th International Conference, ACNS 2017, Kanazawa, Japan, July 10-12, 2017, Proceedings (Lecture Notes in Computer Science), Dieter Gollmann, Atsuko Miyaji, and Hiroaki Kikuchi (Eds.), Vol. 10355. Springer, 557-578. https://doi. org/10.1007/978-3-319-61204-1_28

[9] Shuo Chen, Rui Wang, XiaoFeng Wang, and Kehuan Zhang. 2010. Side-Channel Leaks in Web Applications: A Reality Today, a Challenge Tomorrow. In 31st IEEE Symposium on Security and Privacy, S\&P 2010, 16-19 May 2010, Berleley/Oakland, California, USA. IEEE Computer Society, 191-206. https://doi.org/10.1109/SP 2010.20

[10] Scott E. Coull and Kevin P. Dyer. 2014. Traffic Analysis of Encrypted Messaging Services: Apple iMessage and Beyond. Comput. Commun. Rev. 44, 5 (2014), 5-11. https://doi.org/10.1145/2677046.2677048

[11] George Danezis, Roger Dingledine, and Nick Mathewson. 2003. Mixminion Design of a Type III Anonymous Remailer Protocol. In 2003 IEEE Symposium on Security and Privacy (S\&P 2003), 11-14 May 2003, Berkeley, CA, USA. IEEE Computer Society, 2-15. https://doi.org/10.1109/SECPRI.2003.1199323

[12] George Danezis and Ian Goldberg. 2009. Sphinx: A Compact and Provably Secure Mix Format. In 30th IEEE Symposium on Security and Privacy (S\&P 2009), 17-20 May 2009, Oakland, California, USA. IEEE Computer Society, 269-282. https://doi.org/10.1109/SP.2009.15

[13] Claudia Diaz, Harry Halpin, and Aggelos Kiayias. 2021. The Nym Network: The Next Generation of Privacy Infrastructure. (2021). https://nymtech.net/nymwhitepaper.pdf

[14] Whitfield Diffie and Susan Landau. 2007. Privacy on the Line: The Politics of Wiretapping and Encryption, Updated and Expanded Edition. The MIT Press.

[15] Roger Dingledine and Nick Mathewson. 2006. Anonymity Loves Company Usability and the Network Effect. In 5th Annual Workshop on the Economics of Information Security, WEIS 2006, Robinson College, University of Cambridge, England, UK, fune 26-28, 2006. http://weis2006.econinfosec.org/docs/41.pdf

[16] Roger Dingledine, Vitaly Shmatikov, and Paul F. Syverson. 2004. Synchronous Batching: From Cascades to Free Routes. In Privacy Enhancing Technologies, 4th International Workshop, PET 2004, Toronto, Canada, May 26-28, 2004, Revised Selected Papers (Lecture Notes in Computer Science), David M. Martin Jr. and
Andrei Serjantov (Eds.), Vol. 3424. Springer, 186-206. https://doi.org/10.1007/ 11423409_12

[17] Cynthia Dwork. 2006. Differential Privacy. In Automata, Languages and Programming, 33rd International Colloquium, ICALP 2006, Venice, Italy, fuly 10-14, 2006, Proceedings, Part II (Lecture Notes in Computer Science), Michele Bugliesi, Bart Preneel, Vladimiro Sassone, and Ingo Wegener (Eds.), Vol. 4052. Springer, 1-12. https://doi.org/10.1007/11787006_1

[18] Kevin P. Dyer, Scott E. Coull, Thomas Ristenpart, and Thomas Shrimpton. 2012. Peek-a-Boo, I Still See You: Why Efficient Traffic Analysis Countermeasures Fail. In IEEE Symposium on Security and Privacy, SP 2012, 21-23 May 2012, San Francisco, California, USA. IEEE Computer Society, 332-346. https://doi.org/10.1109/SP. 2012.28

[19] Elixxir. 2019. Elixxir: Architecture Brief. (2019). https://xx.network/elixxirarchitecture-brief-v1.0.pdf

[20] Steven Englehardt, Dillon Reisman, Christian Eubank, Peter Zimmerman, Jonathan R. Mayer, Arvind Narayanan, and Edward W. Felten. 2015. Cookies That Give You Away: The Surveillance Implications of Web Tracking. In Proceedings of the 24th International Conference on World Wide Web, WWW 2015, Florence, Italy, May 18-22, 2015, Aldo Gangemi, Stefano Leonardi, and Alessandro Panconesi (Eds.). ACM, 289-299. https://doi.org/10.1145/2736277.2741679

[21] William Feller. 1968. An introduction to probability theory and its applications: volume I. John Wiley \& Sons New York.

[22] Nethanel Gelernter, Amir Herzberg, and Hemi Leibowitz. 2017. Two Cents for Strong Anonymity: The Anonymous Post-office Protocol. In Cryptology and Network Security - 16th International Conference, CANS 2017, Hong Kong, China, November 30 - December 2, 2017, Revised Selected Papers (Lecture Notes in Computer Science), Srdjan Capkun and Sherman S. M. Chow (Eds.), Vol. 11261. Springer, 390-412. https://doi.org/10.1007/978-3-030-02641-7_18

[23] David M. Goldschlag, Michael G. Reed, and Paul F. Syverson. 1999. Onion Routing. Commun. ACM 42, 2 (1999), 39-41. https://doi.org/10.1145/293411.293443

[24] Benjamin Greschbach, Gunnar Kreitz, and Sonja Buchegger. 2012. The devil is in the metadata - New privacy challenges in Decentralised Online Social Networks. In Tenth Annual IEEE International Conference on Pervasive Computing and Communications, PerCom 2012, March 19-23, 2012, Lugano, Switzerland, Workshop Proceedings. IEEE Computer Society, 333-339. https://doi.org/10.1109/PerComW. 2012.6197506

[25] D.P. Heyman and M.J. Sobel. 2004. Superposition of renewal processes. Stochastic Models in Operations Research: Stochastic Processes and Operating Characteristics (2004).

[26] HOPR. 2021. The Book of HOPR. (2021). https://hoprnet.org/assets/documents/ Book_of_HOPR_v1.pdf

[27] Dogan Kesdogan, Jan Egner, and Roland Büschkes. 1998. Stop-and-Go-MIXes Providing Probabilistic Anonymity in an Open System. In Information Hiding, Second International Workshop, Portland, Oregon, USA, April 14-17, 1998, Proceedings (Lecture Notes in Computer Science), David Aucsmith (Ed.), Vol. 1525. Springer, 83-98. https://doi.org/10.1007/3-540-49380-8_7

[28] Albert Kwon, David Lu, and Srinivas Devadas. 2019. XRD: Scalable Messaging System with Cryptographic Privacy. CoRR abs/1901.04368 (2019). arXiv:1901.04368 http://arxiv.org/abs/1901.04368

[29] Issie Lapowsky. 2018. "Facebook Gave a Russian Internet Giant a Special Data Extension",. $\quad$ https://www.wired.com/story/facebook-gave-russian-internetgiant-special-data-extension/.

[30] Natasha Lomas. 2021. "Nym gets $6 \mathrm{M}$ for its anonymous overlay mixnet to sell privacy as a service". https://techcrunch.com/2021/07/16/nym-gets- $6 \mathrm{~m}$-for-itsanonymous-overlay-mixnet-to-sell-privacy-as-a-service/.

[31] Sandra C Matz, Michal Kosinski, Gideon Nave, and David J Stillwell. 2017. Psychological targeting as an effective approach to digital mass persuasion. Proceedings of the national academy of sciences 114, 48 (2017), 12714-12719.

[32] Jonathan R. Mayer, Patrick Mutchler, and John C. Mitchell. 2016. Evaluating the privacy properties of telephone metadata. Proc. Natl. Acad. Sci. USA 113, 20 (2016), 5536-5541. https://doi.org/10.1073/pnas.1508081113

[33] Michael Mitzenmacher and Eli Upfal. 2005. Probability and Computing: Randomized Algorithms and Probabilistic Analysis. Cambridge University Press. https://doi.org/10.1017/CBO9780511813603

[34] Ulf Möller, Lance Cottrell, Peter Palfrader, and Len Sassaman. 2004. Mixmaster anonymous remailer. http://mixmaster.sourceforge.net/

[35] Steven J. Murdoch. 2006. Hot or not: revealing hidden services by their clock skew. In Proceedings of the 13th ACM Conference on Computer and Communications Security, CCS 2006, Alexandria, VA, USA, October 30 - November 3, 2006, Ari Juels, Rebecca N. Wright, and Sabrina De Capitani di Vimercati (Eds.). ACM, 27-36. https://doi.org/10.1145/1180405.1180410

[36] Steven J. Murdoch and George Danezis. 2005. Low-Cost Traffic Analysis of Tor. In 2005 IEEE Symposium on Security and Privacy (S\&P 2005), 8-11 May 2005, Oakland, CA, USA. IEEE Computer Society, 183-195. https://doi.org/10.1109/SP.2005.12

[37] Laisen Nie, Dingde Jiang, and Zhihan Lv. 2017. Modeling network traffic for traffic matrix estimation and anomaly detection based on Bayesian network in cloud computing networks. Annales des Télécommunications 72, 5-6 (2017), 297-305. https://doi.org/10.1007/s12243-016-0546-3 
[38] Lasse Øverlier and Paul F. Syverson. 2006. Locating Hidden Servers. In 2006 IEEE Symposium on Security and Privacy (S\&P 2006), 21-24 May 2006, Berkeley, California, USA. IEEE Computer Society, 100-114. https://doi.org/10.1109/SP 2006.24

[39] Andriy Panchenko, Fabian Lanze, Jan Pennekamp, Thomas Engel, Andreas Zinnen, Martin Henze, and Klaus Wehrle. 2016. Website Fingerprinting at Inter net Scale. In 23rd Annual Network and Distributed System Security Symposium, NDSS 2016, San Diego, California, USA, February 21-24, 2016. The Internet Society. http://wp.internetsociety.org/ndss/wp-content/uploads/sites/25/2017/09/ website-fingerprinting-internet-scale.pdf

[40] Andriy Panchenko, Asya Mitseva, Martin Henze, Fabian Lanze, Klaus Wehrle, and Thomas Engel. 2017. Analysis of Fingerprinting Techniques for Tor Hidden Services. In Proceedings of the 2017 on Workshop on Privacy in the Electronic Society, Dallas, TX, USA, October 30 - November 3, 2017, Bhavani M. Thuraisingham and Adam J. Lee (Eds.). ACM, 165-175. https://doi.org/10.1145/3139550.3139564

[41] Andriy Panchenko, Lukas Niessen, Andreas Zinnen, and Thomas Engel. 2011 Website fingerprinting in onion routing based anonymization networks. In Proceedings of the 10th annual ACM workshop on Privacy in the electronic society WPES 2011, Chicago, IL, USA, October 17, 2011, Yan Chen and Jaideep Vaidya (Eds.). ACM, 103-114. https://doi.org/10.1145/2046556.2046570

[42] Kyungwon Park and Hyoungshick Kim. 2015. Encryption is Not Enough: Inferring User Activities on KakaoTalk with Traffic Analysis. In Information Security Applications - 16th International Workshop, WISA 2015, Jeju Island, Korea, August 20-22, 2015, Revised Selected Papers (Lecture Notes in Computer Science), Howon Kim and Dooho Choi (Eds.), Vol. 9503. Springer, 254-265. https://doi.org/10.1007/978-3-319-31875-2_21

[43] Ania M. Piotrowska. 2020. Mix Network Simulator. https://github.com/aniampio/ Simulator.

[44] Ania M. Piotrowska, Jamie Hayes, Tariq Elahi, Sebastian Meiser, and George Danezis. 2017. The Loopix Anonymity System. In 26th USENIX Security Symposium, USENIX Security 2017, Vancouver, BC, Canada, August 16-18, 2017, Engin Kirda and Thomas Ristenpart (Eds.). USENIX Association, 11991216. https://www.usenix.org/conference/usenixsecurity17/technical-sessions/ presentation/piotrowska

[45] Robert Potter. [n.d.]. "China is using mass surveillance to impose its will and violate the norms of privacy",. https://www.telegraph.co.uk/opinion/2021/03/ 31/china-using-mass-surveillance-impose-will-violate-norms-privacy/

[46] Mai Schotz. [n.d.]. "Cambridge Analytica Took 50M Facebook Users' Data-And Both Companies Owe Answers",. https://www.wired.com/story/cambridgeanalytica-50m-facebook-users-data/

[47] Robert Kiel Sebastian Burgel. 2019. HOPR - a Decentralized and Metadata-Private Messaging Protocol with Incentives. (2019). https://github.com/hoprnet/hoprwhitepaper

[48] Andrei Serjantov and George Danezis. 2002. Towards an Information Theoretic Metric for Anonymity. In Privacy Enhancing Technologies, Second International Workshop, PET 2002, San Francisco, CA, USA, April 14-15, 2002, Revised Papers (Lecture Notes in Computer Science), Roger Dingledine and Paul F. Syverson (Eds.), Vol. 2482. Springer, 41-53. https://doi.org/10.1007/3-540-36467-6_4

[49] Sandra Siby, Marc Juárez, Claudia Díaz, Narseo Vallina-Rodriguez, and Carmela Troncoso. 2020. Encrypted DNS -> Privacy? A Traffic Analysis Perspective In 27th Annual Network and Distributed System Security Symposium, NDSS 2020, San Diego, California, USA, February 23-26, 2020. The Internet Society. https://www.ndss-symposium.org/ndss-paper/encrypted-dns-privacy-atraffic-analysis-perspective/

[50] Dawn Xiaodong Song, David A. Wagner, and Xuqing Tian. 2001. Timing Analysis of Keystrokes and Timing Attacks on SSH. In 10th USENIX Security Symposium, August 13-17, 2001, Washington, D.C., USA, Dan S. Wallach (Ed.). USENIX. http //www.usenix.org/publications/library/proceedings/sec01/song.html

[51] Paul F. Syverson, Gene Tsudik, Michael G. Reed, and Carl E. Landwehr. 2000 Towards an Analysis of Onion Routing Security. In Designing Privacy Enhancing Technologies, International Workshop on Design Issues in Anonymity and Unobservability, Berkeley, CA, USA, July 25-26, 2000, Proceedings (Lecture Notes in Computer Science), Hannes Federrath (Ed.), Vol. 2009. Springer, 96-114. https://doi.org/10.1007/3-540-44702-4_6

[52] Nirvan Tyagi, Yossi Gilad, Derek Leung, Matei Zaharia, and Nickolai Zeldovich 2017. Stadium: A Distributed Metadata-Private Messaging System. In Proceedings of the 26th Symposium on Operating Systems Principles, Shanghai, China, October 28-31, 2017. ACM, 423-440. https://doi.org/10.1145/3132747.3132783

[53] Jelle van den Hooff, David Lazar, Matei Zaharia, and Nickolai Zeldovich. 2015. Vuvuzela: scalable private messaging resistant to traffic analysis. In Proceedings of the 25th Symposium on Operating Systems Principles, SOSP 2015, Monterey, CA, USA, October 4-7, 2015, Ethan L. Miller and Steven Hand (Eds.). ACM, 137-152. https://doi.org/10.1145/2815400.2815417

\section{A A DIRECT NAME FOR THE APPENDIX}

\section{A.1 Shannon Entropy}

Let $X$ be a discrete random variable over the finite set $X$ with probability mass function $p(x)=\operatorname{Pr}(X=x)$. The Shannon entropy $H(X)$ of a discrete random variable $X$ is defined as

$$
H(X)=-\sum_{x \in X} p(x) \log p(x)
$$

\section{A.2 Sender-Receiver Unlinkability}

The sender-receiver unlinkability metric [44] is defined as follows. Let $I_{1}$ and $I_{2}$ denote events that the sender of the target packet was $S_{1}$ or $S_{2}$ respectively. As $\epsilon$ we denote the measure of information leaked by the system and define it as

$$
\epsilon=\log \left(\frac{\operatorname{Pr}\left(I_{1}\right)}{\operatorname{Pr}\left(I_{2}\right)}\right)
$$

$\epsilon$ is the maximum leakage the adversary can learn from observing the system during events $I_{1}$ and $I_{2}$. Additionally, we denote as $\delta$ the probability by which the leakage exceeds this $\epsilon$.

The above definition quantifies the information leakage in a single round of observation. However, multiple observations increase the adversary's advantage. One way to compute the leakage for multiple rounds is to apply the differential privacy composition theorem [17]. However, this theorem assumes the worst case leakage in each round. Instead, we compute the average case leakage. From the Law of Large numbers [21], we know that for large number of repeated experiments the average of the results converges to the expected value of the outcomes. Hence, in order to compute the anonymity leakage of an observed round $R_{i}$ we compute the value of $\hat{\epsilon}$ for a large number of samples $K$ as follows

$$
\hat{\epsilon}=\frac{1}{K} \sum_{i=1}^{K} \log \left(\frac{\operatorname{Pr}_{i}\left(I_{1}\right)}{\operatorname{Pr}_{i}\left(I_{2}\right)}\right) \text {. }
$$

The overall advantage after $R$ observations can be expressed as $\epsilon_{R}=R \cdot \hat{\epsilon}$ 